\title{
On Sequences of Integers with No 4, or No 5 Numbers in Arithmetical Progression
}

\author{
By S. S. Wagstaff, Jr.
}

Let $k$ be an integer greater than one. We denote by $A^{(k)}(x)$ the maximal number of integers that can be selected from among $1,2, \cdots, x$ to form a set containing no $k$ consecutive terms of an arithmetical progression (no $k$ in a.p.).

Let $\boldsymbol{a}^{(k)}(x)=\left(A^{(k)}(x)\right) / x$.

The function $A^{(k)}(x)$ satisfies a triangle inequality:

$$
A^{(k)}(x+y) \leqq A^{(k)}(x)+A^{(k)}(y),
$$

and, from this, it follows [7] that

$$
\tau^{(k)}=\lim _{x \rightarrow \infty} a^{(k)}(x)
$$

exists.

A well-known conjecture states that, for every $k$, we have

$$
\tau^{(k)}=0 \text {. }
$$

This would imply that every strictly increasing sequence of integers with positive upper density contains arbitrarily long arithmetical progressions. Although the conjecture (2) has been the subject of considerable interest (see references) over the past 30 years, our knowledge in relation to it remains very limited. That $\tau^{(2)}=0$ is trivial. In 1952, K. F. Roth [4], [5] proved that $\tau^{(3)}=0$. The cases of (2) for $k>3$ remain unsettled.

Although the conjecture (2) has proved very resistant, it is not hard in principle to obtain upper bounds for $\tau^{(k)}$ (for any particular $k$ ).

For it is easily seen that (1) implies $\tau^{(k)}=\inf _{x} a^{(k)}(x)$, so that we need only compute $A^{(k)}(x)$ for particular $x$ to obtain upper bounds for $\tau^{(k)}$. The first such computations, in the case $k=3$, were carried out by Erdös and Turán [1] in 1936 (but see also [2] for a correction of these results). More precise results were obtained by L. Moser [3] in 1953. The purpose of the present paper is to obtain upper bounds for $\tau^{(4)}$ and $\tau^{(5)}$ similar to those for $\tau^{(3)}$ before $\tau^{(3)}=0$ was known.

I wish to thank Professor Roth for suggesting the problem to me, and for his help and encouragement in preparing this paper for publication. Thanks also goes to E. Jensen and R. Howell of M.I.T., whose clever ideas helped my computer program run efficiently.

Using the PDP-1 computer at M.I.T., I computed values of $A^{(3)}(x)$ and $A^{(4)}(x)$ for $1 \leqq x \leqq 52$, and of $A^{(5)}(x)$ for $1 \leqq x \leqq 31$. See Table I. The values of $A^{(3)}(x)$ were computed for comparison with the other results.

The best results obtained were

$$
\tau^{(4)} \leqq 1 / 2 \quad \text { and } \quad \tau^{(5)} \leqq 9 / 13 .
$$

Received February 3, 1967. 
TABLE I

\begin{tabular}{|c|c|c|c|c|c|c|}
\hline$x$ & $A^{(3)}(x)$ & $a^{(3)}(x)$ & $A^{(4)}(x)$ & $a^{(4)}(x)$ & $A^{(5)}(x)$ & $a^{(5)}(x)$ \\
\hline 1 & 1 & 1.000 & 1 & 1.000 & 1 & 1.000 \\
\hline 2 & 2 & 1.000 & 2 & 1.000 & 2 & 1.000 \\
\hline 3 & 2 & .667 & 3 & 1.000 & 3 & 1.000 \\
\hline 4 & 3 & .750 & 3 & .750 & 4 & 1.000 \\
\hline 5 & 4 & .800 & 4 & .800 & 4 & .800 \\
\hline 6 & 4 & .667 & 5 & .833 & 5 & .833 \\
\hline 7 & 4 & .571 & 5 & .714 & 6 & .857 \\
\hline 8 & 4 & .500 & 6 & .750 & 7 & .875 \\
\hline 9 & 5 & .556 & 7 & .778 & 8 & .889 \\
\hline 10 & 5 & .500 & 8 & .800 & 8 & .800 \\
\hline 11 & 6 & .545 & 8 & .727 & 9 & .818 \\
\hline 12 & 6 & .500 & 8 & .667 & 10 & .833 \\
\hline 13 & 7 & .538 & 9 & .692 & 11 & .846 \\
\hline 14 & 8 & .571 & 9 & .643 & 12 & .857 \\
\hline 15 & 8 & .533 & 10 & .667 & 12 & .800 \\
\hline 16 & 8 & .500 & 10 & .625 & 13 & .813 \\
\hline 17 & 8 & .471 & 11 & .647 & 14 & .824 \\
\hline 18 & 8 & .444 & 11 & .612 & 15 & .833 \\
\hline 19 & 8 & .421 & 12 & .632 & 16 & .842 \\
\hline 20 & 9 & .450 & 12 & .600 & 16 & .800 \\
\hline 21 & 9 & .429 & 13 & .619 & 16 & .762 \\
\hline 22 & 9 & .409 & 13 & .591 & 16 & .727 \\
\hline 23 & 9 & .391 & 14 & .609 & 16 & .696 \\
\hline 24 & 10 & .417 & 14 & .583 & 17 & .708 \\
\hline 25 & 10 & .400 & 15 & .600 & 18 & .720 \\
\hline 26 & 11 & .423 & 15 & .577 & 18 & .692 \\
\hline 27 & 11 & .407 & 16 & .593 & 19 & .704 \\
\hline 28 & 11 & .393 & 17 & .607 & 20 & .714 \\
\hline 29 & 11 & .379 & 17 & .586 & 21 & .724 \\
\hline 30 & 12 & .400 & 18 & .600 & 21 & .700 \\
\hline 31 & 12 & .387 & 18 & .581 & 22 & .710 \\
\hline 32 & 13 & .406 & 18 & .563 & & \\
\hline 33 & 13 & .394 & 19 & .576 & & \\
\hline 34 & 13 & .382 & 20 & .588 & & \\
\hline 35 & 13 & .371 & 20 & .571 & & \\
\hline 36 & 14 & .389 & 20 & .556 & & \\
\hline 37 & 14 & .378 & 21 & .568 & & \\
\hline 38 & 14 & .368 & 21 & .553 & & \\
\hline 39 & 14 & .359 & 21 & .538 & & \\
\hline 40 & 15 & .375 & 22 & .550 & & \\
\hline 41 & 16 & .390 & 22 & .537 & & \\
\hline 42 & 16 & .381 & 22 & .524 & & \\
\hline 43 & 16 & .372 & 23 & .535 & & \\
\hline 44 & 16 & .364 & 23 & .523 & & \\
\hline 45 & 16 & .356 & 24 & .533 & & \\
\hline 46 & 16 & .348 & 24 & .522 & & \\
\hline 47 & 16 & .340 & 24 & .511 & & \\
\hline 48 & 16 & .333 & 25 & .521 & & \\
\hline 49 & 16 & .327 & 25 & .510 & & \\
\hline 50 & 16 & .320 & 26 & .520 & & \\
\hline 51 & 17 & .333 & 26 & .510 & & \\
\hline 52 & 17 & .327 & 26 & .500 & & \\
\hline
\end{tabular}


In contrast, the best result for $\tau^{(3)}$ was

$$
\tau^{(3)} \leqq 8 / 25 \text {. }
$$

Compare with Moser [3].

An analysis of the maximal deviations of $A^{(4)}(x)$ from $x / 2$ and of $A^{(5)}(x)$ from $9 x / 13$ shows that

$$
A^{(4)}(x) \leqq x / 2+3,
$$

with equality holding for $x=10,28,30,34$, and possibly other values of $x$ congruent to these values $(\bmod 52)$, and

$$
A^{(5)}(x) \leqq 9 x / 13+37 / 13,
$$

with equality holding only for $x=19$, and possibly for $45,71,97$, etc.

The following procedure was used in the computation. Suppose $x$ and $y$ are natural numbers with $y \leqq x$. We can decide whether $y \leqq A^{(k)}(x)$ or $y>A^{(k)}(x)$ by generating all possible selections of $y$ distinct integers from $1,2, \cdots, x$, and testing each selection for $k$ numbers in a.p. If $k$ numbers in a.p. are found in every selection, then $y>A^{(k)}(x)$. If, for some selection, there are no $k$ in a.p., then $y \leqq A^{(k)}(x)$.

It suffices to consider selections

$$
u_{1}, u_{2}, \cdots, u_{y}
$$

such that

$$
1 \leqq u_{1}<u_{2}<\cdots<u_{y} \leqq x .
$$

To test such a selection for $k$ numbers in a.p. effectively, we check each of the numbers $u_{k}, u_{k+1}, \cdots, u_{y}$ for forming an a.p. with $k-1$ of the numbers smaller than it. For each $u_{i}, k \leqq i \leqq y$, this check may be accomplished by considering the possible common differences

$$
d=1,2, \cdots,\left[\left(u_{i}-1\right) /(k-1)\right],
$$

and deciding whether the numbers

$$
u_{i}-d, u_{i}-2 d, \cdots, u_{i}-(k-1) d
$$

are present among the $u_{j}$ for $j<i$. If they are, then the numbers (3) contain the a.p. with highest term $u_{i}$, and common difference $d$.

If, for some integer $m$, it is known that $A^{(k)}(m)=n$, then $A^{(k)}(m+1)$ can only be $n$ or $n+1$. Let $x=m+1$, and $y=n+1$. Then the above procedure tells us whether $n+1 \leqq A^{(k)}(m+1)$, or $n+1>A^{(k)}(m+1)$, i.e., whether $n+1=$ $A^{(k)}(m+1)$, or $n=A^{(k)}(m+1)$. In this manner $A^{(k)}(x)$ can be easily computed for consecutive natural numbers $x$.

Suppose $x$ is chosen, $A^{(k)}(i)$ are known for $1 \leqq i<x$, and we want to compute $A^{(k)}(x)$. Then we set $y=1+A^{(k)}(x-1)$, and apply the above procedure. The sequences (3) satisfying (4) must also satisfy

$$
i \leqq u_{i} \leqq x-y+i, \text { for } 1 \leqq i \leqq y .
$$

For each natural number $n<y$, let $B^{(k)}(n)$ be the smallest number $m$ such that 
$n=A^{(k)}(m)$. Let $B^{(k)}(0)=0$, and $B^{(k)}(y)=1+B^{(k)}(y-1)$. Then we can improve the bounds on $u_{i}$ in (5) to

$$
B^{(k)}(i) \leqq u_{i} \leqq x-B^{(k)}(y-i) .
$$

This narrowing of the bounds on $u_{i}$ significantly reduces the number of sequences to be considered, and hence the computer running time. The computation of $A^{(4)}(x)$ for $x>40$ would not have been feasible on the PDP-1 without these improved bounds.

In the actual program, the upper bound in (6) was implemented, but the lower bound proved too difficult to program, so I used another technique. The numbers $1,2, \cdots, y$ were deposited in $y$ consecutive registers. This was the first sequence considered. Each sequence was tested as follows. First the $k$ th number was checked for being the highest of $k$ numbers in a.p., then the $(k+1)$ th number was so checked, then the $(k+2)$ th, etc., to the $y$ th number. This check was performed essentially as above. The checking process stops as soon as an a.p. is found. If one is found, let the $j$ th number be that which was being tested, i.e., the highest number in the a.p. found. If no a.p. is found, the sequence is typed, and we let $j=x$. Now let $i$ be the largest number such that $i \leqq j$, and the $i$ th number is not $x-B^{(k)}(y-i)$. If no such number $i$ exists, then all sequences which could possibly have no $k$ in a.p. have been examined, so the program halts. Otherwise, let $m$ be the $i$ th number. Then the modification made to the current sequence to produce the next one is to deposit the numbers

$$
m+1, m+2, \cdots, m+y-i+1
$$

into the $i$ th, $(i+1)$ th, $\cdots, y$ th registers. If the program types any sequences, we know that $y \leqq A^{(k)}(x)$, and if none are printed, $y>A^{(k)}(x)$.

Table II

$k=4, x=8, y=6$

$\begin{array}{llllll}1 & 2 & 3 & 4 & 5 & 6 \\ 1 & 2 & 3 & \overline{5} & 6 & 7 \\ 1 & 2 & 3 & 5 & 6 & \overline{8}^{*} \\ 1 & 2 & 3 & 5 & 7 & \overline{8}^{2} \\ 1 & 2 & 3 & 6 & \frac{7}{7} & 8^{*} \\ 1 & 2 & 4 & 5 & 6 & 7 \\ 1 & 2 & 4 & 5 & 6 & \overline{8} \\ 1 & 2 & 4 & 5 & 7 & \overline{8}^{*} \\ 1 & 2 & 4 & 6 & 7 & \overline{8}^{8} \\ 1 & 3 & 4 & 5 & 6 & 7 \\ 1 & 3 & 4 & 5 & 7 & 8 \\ 1 & 3 & 4 & 6 & \frac{7}{7} & 8^{*} \\ 2 & 3 & 4 & 5 & 6 & 7 \\ 2 & 3 & 4 & 6 & 7 & 8\end{array}$

HALT.

\footnotetext{
* Indicates sequences typed.
} 
For an example of the above procedure, see Table II. The $j$ th number in each sequence is underlined. The starred sequences are those typed. Note that only 14 sequences need be considered, while there are $C_{8,6}=28$ sequences that satisfy (4) and (5).

Sequences were typed on-line, and, for $x<20$, running time was negligible compared to typing time. The cycle time of the PDP-1 is 5 microseconds. For large $x$, the program ran for several hours. To save time, the program was stopped after one or two sequences were typed, for certain large $x$. Also, for certain small $x$, when it was clear that a large number of sequences would be typed, the program was stopped after 20 or 30 of them were typed.

Cornell University

Ithaca, New York 14850

1. P. Erdös \& P. Turán, "On some sequences of integers," Proc. London Math. Soc. (2), v 11, 1936, pp. 261-264.

2. A. Makowski, "Remark on a paper of Erdös and Turán," J. London Math. Soc., v. 34, 1959, p. 480. MR 21 \#5596.

3. L. Moser, "On non-averaging sets of integers," Canad. J. Math. v. 5, 1953, pp. 245-252. MR 14, 726.

4. K. F. Rотн, "On certain sets of integers," J. London Math. Soc., v. 28, 1953, pp. 104-109. MR 14, 536.

5. K. F. Roth, "Sur quelques ensembles d'entiers," C. R. Acad. Sci. Paris, v. 234, 1952, pp. 388-390. MR 13, 724.

6. R. Salem \& D. C. Spencer, "On sets of integers which contain no three terms in arithmetic progression," Proc. Nat. Acad. Sci. U.S.A., v. 28, 1942, pp. 561-563. MR 4, 131.

7. R. Salem \& D. C. Spencer, "On sets which do not contain a given number of terms in arithmetic progression," Nieuw Arch. Wiskunde (2), v. 23, 1950, pp. 133-143. MR 11, 417. 